\title{
Effect of Bose-Einstein correlations on intermittency in ultrarelativistic nucleus-nucleus collisions
}

\author{
F. Kun ${ }^{\text {a,1 }}$, H. Sorge ${ }^{\text {b }}$, K. Sailer ${ }^{\text {a }}$, M. Hofmann ${ }^{\text {b }}$, G. Bardos ${ }^{\text {a }}$, W. Greiner ${ }^{b}$ \\ ${ }^{a}$ Department of Theoretical Physics, Kossuth Lajos University, H-4010 Debrecen, P.O. Box 5., Hungary \\ b Institut für Theoretische Physik, Johann Wolfgang Goethe Universität, D-6000 Frankfurt am Main, Postfach 111932, Germany
}

Received 9 February 1994

Editor: R. Gatto

\begin{abstract}
The role of quantum correlations, the rescattering of the produced particles and mixing of events with various impact parameters on intermittency in nucleus-nucleus collisions at $200 \mathrm{~A} \mathrm{GeV}$ is studied in the framework of the RQMD model. We generated an RQMD data sample with Bose-Einstein correlations of identical pion pairs included. One can get quantitative agreement with the measured intermittent behaviour of the pion pseudorapidity spectra if these quantum correlations are taken into account.
\end{abstract}

\section{Motivations}

To investigate properties of dynamical fluctuations in relativistic heavy ion collisions Bialas and Peschanski proposed to study the scaled factorial moments of the (pseudo) rapidity distribution of the final state particles and introduced the concept of intermittency into high energy nuclear physics [1]. The effect of intermittency is quantified by the power law behaviour of the scaled factorial moments $\left(F_{i}\right)$ and loosely regarded as having fractal properties.

Intermittent behaviour of spectra was observed in several experiments. After an early analysis of cosmic-ray events this effect has been observed in $e^{+} e^{-}$, muon-hadron, hadron-hadron, hadron-nucleus and nucleus-nucleus collisions [2], thus it seems to

\footnotetext{
${ }^{1}$ E-mail: KUN@HUKLTE51.BITNET. Fax: 36-52-416181.
}

be a universal feature of particle production at high energies.

It is an important question whether the intermittency can be understood on the basis of our conventional physical knowledge or we should suspect new physics behind it. For answering this question it is necessary to study whether the commonly used models of particle production can account for the general features of the observed intermittent behaviour. This problem was studied using Fritiof, Jetset, Herwig, Pythia and Venus codes which generally give a good description of the single particle features observed in experiments. However, it was found that intermittency effects are much stronger in the measured data than in these Monte Carlo simulations. It means quantitatively that the anomalous fractal dimensions $\left(d_{i}\right)$ calculated from the experimental data are several times larger than those calculated from the models, otherwise, practically there was no intermittency $\left(d_{i} \sim 0\right)$ in several cases in the model calculations $[2,8]$. Fur- 
ther checks are of course necessary to understand this situation.

It is a well-known fact that in the experiments the impact parameter of the collisions cannot be identified precisely, the analysis of events from experiments naturally includes mixing events of widely different impact parameters. Recently the effect of mixing events on the factorial moments was investigated by $\mathrm{Li}$ using the Boltzmann-Uehling-Uhlenbeck approach $[9,10]$ for ${ }^{16} \mathrm{O}+\mathrm{Ag} / \mathrm{Br}$ collisions at $2.1 \mathrm{GeV} /$ nucleon energy. It was found that the values of anomalous fractal dimensions are practically insensitive to the impact parameter $b$, only the value of the moments has $b$ dependence. The averaged multiplicity of the event sample was very small that can result in a rather big error in the calculation of the factorial moments and anomalous fractal dimensions. So, it is worth reconsidering this problem in systems where the multiplicities are higher.

The transport models of heavy ion reactions are based on classical propagation between stochastic collision and decay points. They do not include quantum mechanical interference effects of a many-body wave function for identical particles. The question naturally arises whether these quantum mechanical effects can give rise to changes in the properties of factorial moments and fractal dimensions calculated from the models.

Our aim here is to study both these problems outlined above within the framework of the RQMD model [11]. $R Q M D$ is a microscopic phase space approach based on resonance and string excitations, fusion of neighboring strings into so-called ropes and subsequent reinteractions of all secondaries with each other and with the original ingoing baryons (see [12] and [13] for a description of the recently updated version RQMD 1.08 which was used for the calculations presented here). In order to study the effect of BoseEinstein correlations on intermittency we have to put the correlation of identical particles due to quantum mechanical symmetry by hand into RQMD, because quantum correlations are not dynamically included. Using the spacetime and momentum coordinates of the particles at their point of last interaction (freezeout) from RQMD we calculated the correlation function of identical pion pairs and generated 'improved' RQMD events which contain the quantum statistical correlation. Both sets of events were analised with the method of factorial moments. We also discuss in this paper whether dynamically generated correlations contribute to intermittency. In the model fluctuations from event to event can be caused by the initialization and random hadron-hadron collisions during the collisional process. For our treatment a major advantage of the RQMD model is that it includes the rescattering of the produced particles, so we could also study whether this rescattering can produce dynamical fluctuations.

Recently, Sengupta et al. studied dynamical fluctuations in quasicentral events from the EMU-08 experiment [ 8 ] performed at the CERN SPS for ${ }^{32} \mathrm{~S}$ beam at $200 \mathrm{GeV} /$ nucleon energy interacting in $\mathrm{Ag} / \mathrm{Br}$ emulsion. We have investigated this reaction in the framework of the RQMD model.

\section{Scaled factorial moments}

The dynamical fluctuations can be described by studying the scaled factorial moments $\left(F_{i}\right)$ [1] of the (pseudo) rapidity distribution of the produced particles:

$$
F_{i}=M^{i-1} \frac{\left\langle\sum_{j=1}^{M} n_{j}\left(n_{j}-1\right) \cdots\left(n_{j}-i+1\right)\right\rangle}{n(n-1) \cdots(n-i+1)}
$$

where the considered pseudorapidity interval $\Delta \eta$ was split into $M$ equal bins of size $\delta \eta=\Delta \eta / M, n_{j}$ is the multiplicity of the $j$-th bin, $n$ is the averaged multiplicity of events and the brackets denote the average over the events of the sample. The power law behaviour of the scaled factorial moments with increasing resolution is called intermittency, namely, $F_{i} \sim(\delta \eta)^{-f_{i}}$ (or $F_{i} \sim M^{f_{i}}$ ) with decreasing $\delta \eta$.

The intermittency indices $\left(f_{i}\right)$ are related to the socalled anomalous fractal dimensions $\left(d_{i}\right): d_{i}=f_{i} /(i-$ $1)$. The analysis of scaled factorial moments reveals properties of fluctuations through both the magnitude of the moments and the slopes of the $\ln F_{i}$ 's. It is easy to show that the factorial moments are independent from the resolution for Poissonian distribution of $n_{j}[14,15]$, i.e. when only statistical fluctuations are present in the system. The large magnitude of the moments requires a sample of events having different multiplicities, and the non-zero slopes of the $\ln F_{i}$ require events having holes or spikes at different positions in the distribution. If one of the intermittency 
indices is non-vanishing it reveals a rich structure of holes and spikes. Intermittency and scaling of particle rapidity distributions may occur as a result of a self-similar cascading process or in a system undergoing second-order phase transition close to the critical point. Such a system is known to scale at the critical point because the correlation length diverges. The correlation functions exhibit power law singularities and this implies the scaling law. In this particular case the intermittency exponents are all related to critical exponents of the phase transition. Consequently, all anomalous fractal dimensions are equal, i.e. the spectrum is described by a mono-fractal. This was demonstrated explicitly for the Ising model $[4,5]$ and for the Feynman-Wilson fluid [3]. There were some efforts to understand the intermittency observed in heavy ion collisions on the basis of the conventional short-range correlation [6,7] and the linked-pair ansatz for higher-order correlations. Good agreement was found between the theory and measurements at lower rank of the factorial moments $(i=2,3)$ for the NA22 experiment. There are also some indications for the connection between the Bose-Einstein correlation and the intermittency [18-21]. This draws attention to the role of the short-range correlations in the factorial moments. Consequently, it is very important to study whether the anomalous fractal dimensions observed in heavy ion reactions can be understood by taking into account the quantum correlations.

\section{Computational procedures, results}

Our method of treatment is briefly the following. At first we generated a sample of events with $R Q M D$ for the heavy ion reaction considered, ${ }^{32} \mathrm{~S}+\mathrm{Ag} / \mathrm{Br}$ with a beam energy of $200 \mathrm{~A} \mathrm{GeV}$. The sample of events on emulsion was composed of elementary collisions between the sulphur projectile and the components of the target, their numbers within the sample were chosen to be proportional to the total crosssections. The data sample contained 1000 events for every fixed impact parameter $b(1,3,5 \mathrm{fm})$, and the number of events was chosen proportional to $b$ for impact parameter mixing, so it was 500,1500 and 2500 for $1,3,5 \mathrm{fm}$ respectively. We analized the calculated pseudorapidity distribution for the factorial moments taking into account mixing of events with var-

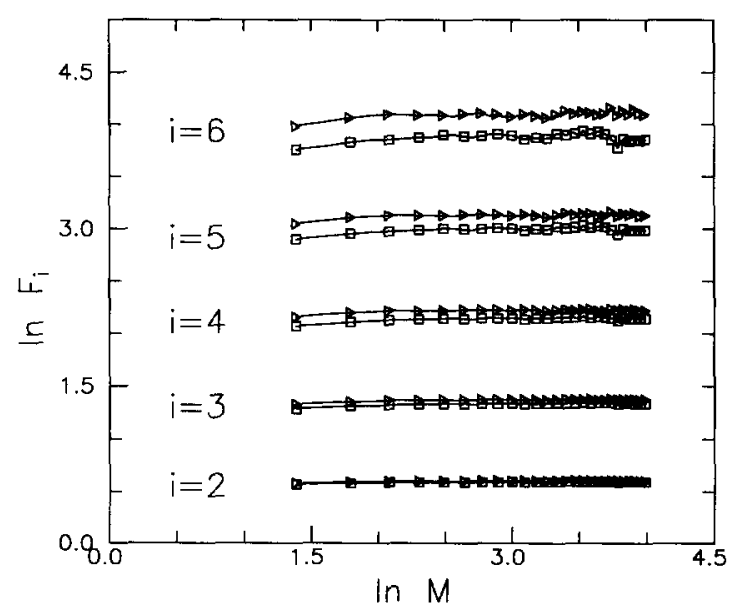

Fig. 1. The calculated scaled factorial moments $F_{i}$ vs. the number of bins $M$ at the impact parameters of $b=1 \mathrm{fm}(\square)$ and $b=3$ fm $(\triangleright) . i$ denotes the rank of the moments.

ious impact parameters. The analysis was performed for charged pions in the $0.5 \leq \eta \leq 5.5$ pseudorapidity window. In a second step we constructed a modified sample of events, containing the quantum statistical two-particle correlation for identical pions. RQMD yields the $\left(x_{i}, p_{i}\right)$ final spacetime and momentum space production points of all the particles where $x_{i}=\left(t_{i}, \boldsymbol{x}_{i}\right), p_{i}=\left(E_{i}, \boldsymbol{p}_{i}\right)$. Using these production points we calculated the pair-correlation caused by Bose-Einstein statistics in the plane-wave approximation [17]. It has already been demonstrated that $\mathrm{RQMD}$ reproduces the measured two-pion correlation in $A A$ collisions at $200 A \mathrm{GeV}$ very well if the quantum statistical two-particle correlation after freeze-out is included (see e.g. Ref. [16]). Afterwards, we modified our data sample to include this calculated paircorrelation, retaining the one-particle pseudorapidity distribution and the multiplicities. Then, the modified sample was reanalized and compared to the experimental data.

Fig. 1 and Fig. 2 show the factorial moments $F_{i}$ vs. the number of bins $M$ for the original RQMD events, i.e. without any Bose-Einstein correlations, for 1,3 $\mathrm{fm}$ and mixed events, respectively. It can be seen that the $F_{i}$ 's are practically independent from the resolution $M$. This holds true even for impact parameter mixed events. It means that only statistical fluctuations are present in the system, the rescattering of the produced particles does not generate dynamical fluc- 


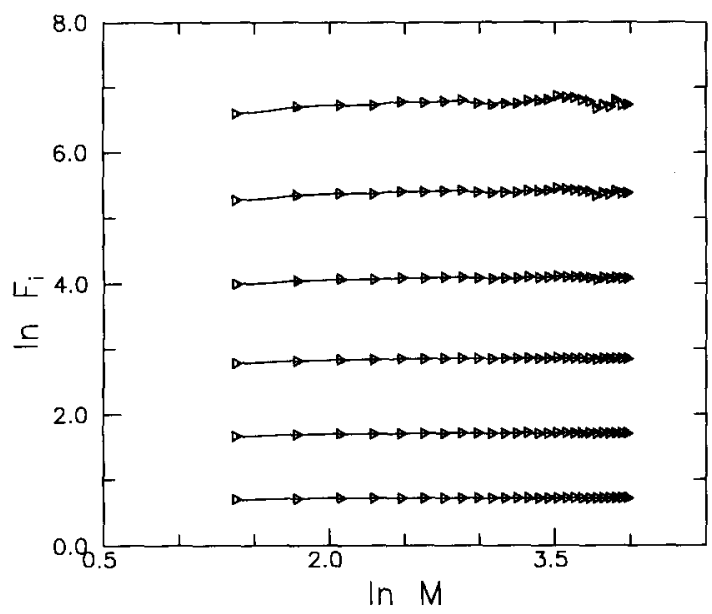

Fig. 2. The scaled factorial moments $F_{i}$ for mixing of events. $(i=2, \ldots, 7)$.

tuations. The anomalous fractal dimensions are practically zero $d_{i} \sim 0$ independently of $b$, but the values of the factorial moments are sensitive to the impact parameter, the increasing $b$ causes increment in $F_{i}$. As expected the value of $F_{i}^{\prime} s$ for mixed events is higher than that for any fixed impact parameter.

The one-particle pseudorapidity distribution and the pseudorapidity pair-correlation function of the original RQMD simulated data set was calculated at the resolution corresponding to the finest resolution in the determination of the factorial moments $(M=100)$. Based on this one-particle pseudorapidity distribution, the multiplicity distribution and the calculated pair-correlation function of the original data set we generated a new data sample in the following way. Every original event with multiplicity $N$ was substituted by $N / 2$ pairs. The centre of a pair was positioned according to the original one-particle distribution, and the difference of the two particle pseudorapidities was stochastically chosen on the basis of the calculated pair correlation. In the new sample created in this way the one-particle distribution and the distribution of event multiplicities remained the same but a strong short-range correlation occurred corresponding exactly to the calculated correlation caused by Bose-Einstein statistics. This new sample was reanalized by means of factorial moments.

Fig. 3 and Fig. 4. show the new factorial moments $F_{i}$ for $b=\mathbf{3} \mathrm{fm}$ and for mixed events as a function of the number of bins $M$. It can be seen that the features

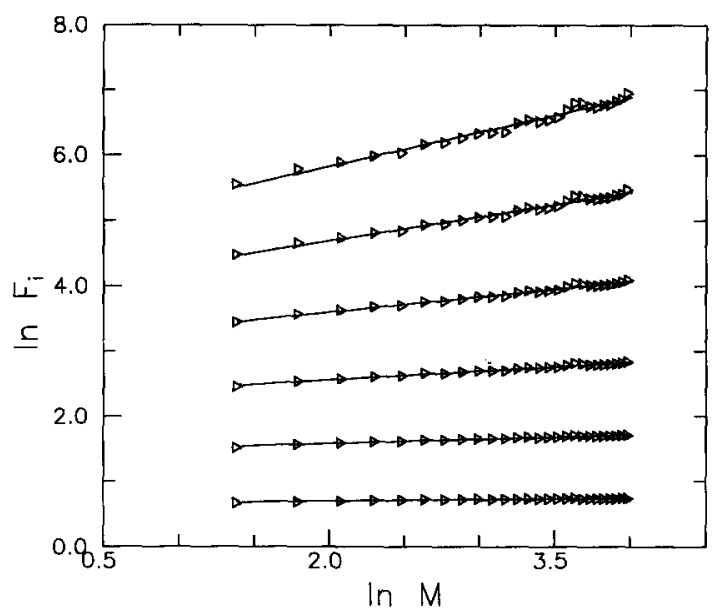

Fig. 3. The calculated scaled factorial moments $F_{i}(i=2, \ldots, 7)$ of the modified sample at $b=3 \mathrm{fm}$. The solid lines are the least square fitted straight lines whose slopes are the intermittency indices $f_{i}$.

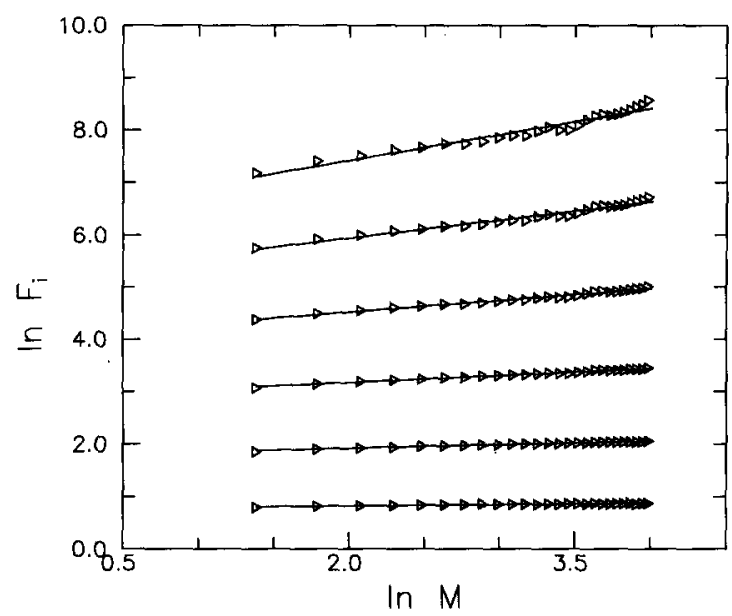

Fig. 4. The scaled factorial moments $F_{i}(i=2, \ldots, 7)$ obtained from the modified sample by mixing of events. The solid lines are the least square fitted straight lines.

of the factorial moments drastically changed, they can be very well approximated by straight lines, and they show characteristic intermittent behaviour. From the slope of the least square fitted straight lines we extracted the anomalous fractal dimensions. Fig. 5 shows the comparison of the anomalous fractal dimensions extracted from the experiments and the new data samples, separately for each impact parameter and for mixed events. It is important to note that the values of $d_{i}$ depend on $b$, increasing $b$ results in larger $d_{i}$. The 


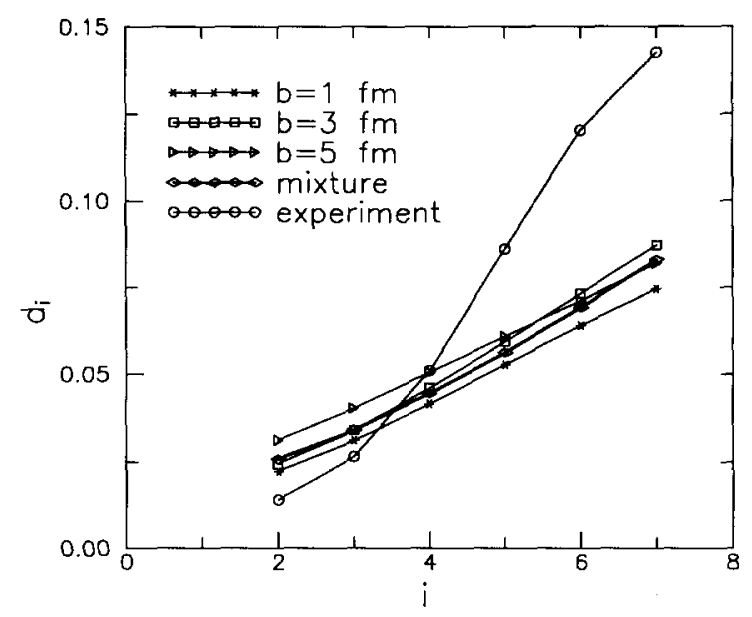

Fig. 5. The anomalous fractal dimensions $d_{i}$ obtained from the experiment and from the modified data samples as a function of the rank of the factorial moments.

mixing of events leads to $d_{i}$ values in an intermediate place. The $d_{i}$ functions can be well approximated by straight lines. The role of the different impact parameters in the values of the factorial moments is the same as before. One can conclude that the quantum correlations have a very strong effect on the values of $d_{i}$. The new anomalous fractal dimensions $d_{i}$ fall close to the measured values, a reasonable quantitative agreement was found.

\section{Conclusions}

In the present paper we have investigated the role of quantum correlations, rescattering of the produced particles and the mixing of events with different impact parameters on the phenomenon of intermittency. It was found that rescattering of the produced particles does not lead to dynamical correlations, and mixing of events has only a weak effect on the factorial moments. Pair correlations have been introduced into the model on the basis of the calculated quantum statistical pair correlation function. This crude incorporation of quantum statistical effects leads already to a relatively good quantitative agreement of the calculated anomalous fractal dimensions with experimental data. It turns out that accounting for quantum statistical effects in the models of heavy-ion reactions is of partic- ular importance in order to obtain a better understanding of intermittency in nucleus-nucleus collisions.

\section{Acknowledgement}

One of the authors (F. Kun) is very grateful to Ch.R. Hofmann and R. Mattiello for the valuable discussions. This work was supported by the Hungarian National Scientific Research Fond (OTKA 316-1313) and the joint project of the Deutsche Forschungsgemeinschaft and the Hungarian Academy of Sciences (№ 43). F. Kun was partly supported by the Universitas Foundation of the Hungarian Credit Bank.

\section{References}

[1] A. Bialas, R. Peschanski, Nucl. Phys. B 273 (1986) 703

[2] A. Bialas, Nucl. Phys. A 525 (1991) 613

[3] N.G. Antoniou et al., CERN preprint TH 5625 (January 1990)

[4] H. Satz, Nucl. Phys. B 326 (1989) 613

[5] B. Bamback, J. Fingberg and H. Satz, Nucl. Phys. B 332 (1990) 629

[6] P. Carruthers, I. Sarcevic, Phys. Rev. Lett. 63 (1989) 1562

[7] I. Sarcevic, Nucl. Phys. A 525 (1991) 361

[8] K. Sengupta, P.L. Jain, G. Singh and S.M. Kim, Phys. Lett. B 236 (1990) 219

[9] B.A. Li, Phys. Lett. B 292 (1992) 246

[10] B.A. Li, Phys. Rev. C 47 (1993) 693

[11] H. Sorge, H. Stöcker and W. Greiner, Ann. Phys. (USA) 192 (1989) 266; Nucl. Phys. A 498 (1989) 567c

[12] H. Sorge, M. Berenguer, H. Stöcker and W. Greiner, Phys. Lett. B 289 (1992) 6

[13] H. Sorge, L. Winckelmann, H. Stöcker and W. Greiner, Z. f. Phys. C 59 (1993) 85

[14] G. Bardos and F. Kun, Phys. Lett. A 165 (1992) 401

[15] A.R. DeAngelis, D.H.E. Gross and R. Heck, Nucl. Phys. A 537 (1992) 606

[16] J.P. Sullivan, M. Berenguer, B.V. Jacak, S. Pratt, M. Sarabura, J. Simon-Gillo, H. Sorge and H. van Hecke, Phys. Rev. Lett. 70 (1993) 3000

[17] Zs. Schram, K. Sailer, B. Müller and W. Greiner, Z. Phys. C 47 (1990) 137

[18] I. Derado, G. Jancso and N. Schmitz, Z. Phys. C 56 (1992) 553

[19] P. Carruthers, E.M. Friedlander, C.C. Shih and R.M. Weiner, Phys. Lett. B 222 (1989) 487

[20] D. Seibert, Phys. Rev. Lett. 63 (1989) 136

[21] I.V. Andreev, M. Plümer, B.R. Schlei and R.M. Weiner, Phys. Lett. B 316 (1993) 583 